Rechtsgebieten lehnt Selbert ab, weil sie darin den Mann als Maßstab der Rechte und Pflichten und die Frau als hiervon abgeleitet erkennt. Das zähe Ringen geht weiter, sie gibt nicht auf, muss aushalten, dass ihr von Theodor Heuss spöttisch die Kompetenz abgesprochen wird, der die Protestwelle als „Quasi-Stürmlein " kleinreden will. Aber am Ende setzt sie sich durch: Am 18. Januar 1949 wird Artikel 3 Absatz 2 GG in dem von ihr vorgeschlagenen Wortlaut ohne jede Gegenstimme angenommen. Einen Tag später wendet sie sich in einer eindrucksvollen Rundfunkansprache an die Bevölkerung:

„... dieser Tag war ein geschichtlicher Tag, eine Wende auf dem Wege der deutschen Frauen der Westzonen. Lächeln Sie nicht! Es ist nicht falsches Pathos einer Frauenrechtlerin, das mich so sprechen läßt. Ich bin Jurist und unpathetisch und Frau und Mutter und zu solchen frauenrechtlerischen Dingen gar nicht geeignet..."

Auch in der Folgezeit mag sie sich nicht auf Frauenfragen festlegen lassen. Im Unterschied zu den anderen drei „Müttern “ des Grundgesetzes scheitert ihr Einzug in den Ersten Deutschen Bundestag, wenn auch knapp. Sie erhält 1956 das Große Bundesverdienstkreuz, bleibt noch bis 1958 in der Landespolitik, konzentriert sich danach aber auf ihre Kanzlei und gerät in der Öffentlichkeit in Vergessenheit. Adam Selbert stirbt im Jahr 1968. Bis über das achtzigste Lebensjahr hinaus geht sie mit großem Einsatz ihrer anwaltlichen Arbeit nach. Am 9. Juni 1986 verstirbt sie in ihrer Heimatstadt Kassel wenige Monate vor ihrem 90. Geburtstag.

„Ich hatte einen Zipfel der Macht in meiner Hand gehabt und den habe ich ausgenutzt, in aller Tiefe, in aller Weite, die mir rhetorisch zur Verfügung stand. Es war die Sternstunde meines Lebens, als die Gleichberechtigung der Frau damit zur Annabme kam."

\section{Literatur}

Böttger, Barbara: Das Recht auf Gleichheit und Differenz. Elisabeth Selbert und der Kampf der Frauen um Art. 3 II Grundgesetz, Münster, 1990; Dertinger, Antje: Elisabeth Selbert. Eine Kurzbiographie, Wiesbaden 1986; dies.: Männer und Frauen sind gleichberechtigt. Die Juristin Elisabeth Selbert erstritt Art. 3 II, in: dies.: Frauen der ersten Stunde. Aus den Gründungsjahren der Bundesrepublik, Bonn 1989; S. 179-190; Drummer, Heike/Zwilling, Jutta: Elisabeth Selbert. Eine Biographie, in: Hessische Landesregierung (Hrsg.): „Ein Glücksfall für die Demokratie“. Elisabeth Selbert (1896-1986). Die große Anwältin der Gleichberechtigung, Frankfurt/Main 1999; Mundzeck, Heike: Elisabeth Selbert, in: Deutscher Juristinnenbund (Hrsg.): Juristinnen in Deutschland. Die Zeit von 1900 bis 1998, Baden-Baden 1998, S. 18-194; Nachlass Elisabeth Selbert, Stiftung Archiv der deutschen Frauenbewegung, Kassel; Notz, Giesela: Frauen in der Mannschaft. Sozialdemokratinnen im Parlamentarischen Rat und im Deutschen Bundestag 1948/49 bis 1957. Bonn 2003, S. 16 ff.; Röwekamp, Marion: Juristinnen - Lexikon zu Leben und Werk, Baden Baden 2005, S. 395-399.

\title{
„Erzählte Geschichte“ in Frauenverbänden
}

\section{Ein Projekt der Stiftung Archiv der deutschen Frauenbewegung}

Der Deutsche Juristinnenbund wurde 1948 gegründet, er geht ursprünglich auf den Deutschen Juristinnen-Verein von 1914 zurück, ist also inzwischen 60 Jahre alt bzw. ideell sogar schon fast 100 Jahre - eine lange Geschichte. Je länger die Geschichte eines Verbandes währt, um so mehr stellt sich die Frage nach ihrer Überlieferung. Doch wie entsteht Überlieferung? Woraus bildet sich das, was schließlich als „die Verbandsgeschichte“ in die Annalen eingeht?

Zum einen sicherlich aus den Akten, die im Laufe der Jahre und Jahrzehnte entstehen (und hoffentlich aufbewahrt werden!). Darin finden sich die Korrespondenzen, die Protokolle der Mitgliederversammlungen und der Sitzungen von Vorständen, Kommissionen und Arbeitsgruppen, Presseerklärungen, Petitionen und andere Verlautbarungen und schließlich auch Unterlagen über die finanzielle Seite wie Bilanzen, Kassen- und Kontoaufstellungen und vieles mehr. Hier finden wir also in erster Linie die schriftliche Überlieferung und - wenn es gut geht - auch eine bildliche in Form von Fotos oder gar Filmen, die zu besonderen Anlässen gemacht und aufbewahrt wurden. All dies wird gelegentlich ergänzt durch Sammlungen einzelner Protagonistinnen des Verbandes, die diese möglicherweise dem Verbandsarchiv übergeben oder hinterlassen.

Ein weiterer, oft vernachlässigter Bereich der historischen Überlieferung sind die persönlichen Erinnerungen der Mitglieder, die es zum Beispiel in Form von Tagebuchaufzeichnungen, schriftlicher Niederlegung der Erinnerungen und in Form von Interviews gibt. Sie ergänzen die vorgenannten, eher sachlichen Belege und beleben sie auch in gewisser Weise. „Erzählte Geschichte“ ist immer eine höchst subjektive Sichtweise einzelner Menschen, die aber dennoch, richtig abgewägt und eingeord-

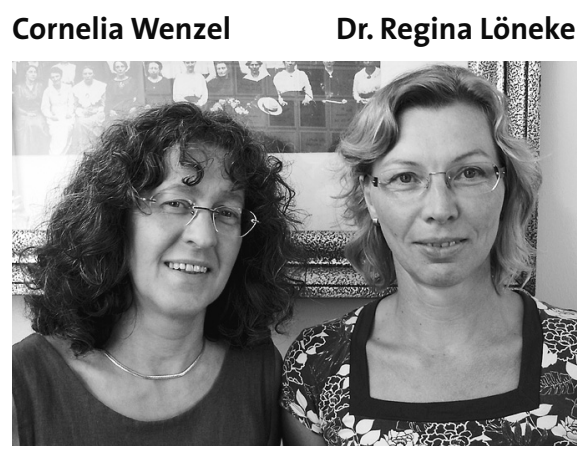

Archiv der deutschen Frauenbewegung, Kassel 
net, das Gesamtbild der Überlieferung ergänzen und anschaulicher machen kann. Die Atmosphäre, die Stimmungen, die leisen Zwischentöne, die kaum schriftlich festgehalten werden, sind hierüber zu vermitteln. Und nicht selten werden über diesen Weg auch Informationen über alles, was aus verschiedensten Gründen nicht im Protokoll festgehalten wurde, weitergegeben und für die Nachwelt erhalten. Gute Gründe also, Frauen, die schon lange Mitglied sind, die die Entwicklung des Verbandes miterlebt und mitgestaltet haben, zu interviewen und diese persönlichen Erinnerungen festzuhalten.

Genau das geschieht seit einiger Zeit in einem Projekt auf Initiative des Archivs der deutschen Frauenbewegung. Anhand von vier Frauenverbänden sollen mit Methoden der Oral History beispielhaft Interviews durchgeführt, anschließend die Arbeitsweise erläutert und zur Nachahmung angeboten werden. Das Projekt steht in einem größeren Zusammenhang: seit dem Jahr 2005 bemüht sich die Stiftung Archiv der deutschen Frauenbewegung, unterstützt vom Bundesministerium für Familie, Senioren, Frauen und Jugend (BMFSFJ) darum, den im Deutschen Frauenrat zusammen geschlossenen Frauenorganisationen das Thema Überlieferungsbildung näher zu bringen, weil sich gezeigt hat, dass viele Verbände nur über sehr lückenhafte Archive verfügen. In diesem Zusammenhang entstand 2005/2006 die Internetpräsentation www.FrauVerA.de mit Tipps und Hinweisen zur Archivierung von Verbandsunterlagen. Im November 2006 folgte dann das Interviewprojekt „Bewahren von Erinnerungen - Frauenverbandsarbeit als Motor für politische Teilhabe und Chancengerechtigkeit: Vier Frauenverbände als Beispiel“. Die vier beteiligten Verbände sind der Deutsche Evangelische Frauenbund (DEF), der Deutsche Juristinnenbund (djb), der Deutsche StaatsbürgerinnenVerband (DStV) und der Katholische Deutsche Frauenbund (KDFB). Aus jedem Verband wurden exemplarisch sieben bis neun Frauen zu ihrer persönlichen Erinnerung der Verbandsgeschichte befragt. Damit entsteht natürlich keineswegs ein vollständiges Bild. Um die Geschichte der jeweiligen Verbände auch nur einigermaßen repräsentativ erfassen zu können, müssten sehr viel mehr Verbandsmitglieder einbezogen werden. Vollständigkeit ist jedoch nicht das Ziel unseres Projektes. Wir wollen vielmehr mit diesen Beispielen aus vier Frauenverbänden mit sehr unterschiedlichen thematischen und organisatorischen Hintergründen beispielhaft erproben, wie sich Methoden der Oral History in diesem Zusammenhang bewähren und was dabei besonders zu beachten ist.

\section{Das Beispiel Juristinnenbund}

Zunächst wurde das Projekt in einem Gespräch der Geschäftsführerin des djb, Anke Gimbal, in der Bundesgeschäftsstelle in Berlin vorgestellt und um Unterstützung bei der Eruierung von Interviewpartnerinnen gebeten. Von der Geschäftsstelle wurde eine erste Zusammenstellung in Frage kommender Verbandsmitglieder erstellt; Frau Gimbal übernahm auch die ersten Anfragen bei den betreffenden Zeitzeuginnen. Weitere Interviewpartnerinnen kamen durch Vorschläge der Rechtsanwältin Martina Bosch (Bonn), Geschäftsführerin des djb bis
2001, hinzu, die darüber hinaus Hintergrundinformationen aus den Beständen des von ihr betreuten online-Verbandsarchivs zur Verfügung stellte. So konnten zwischen Mitte 2007 und Anfang 2008 neun Interviews mit acht Mitgliedern des Juristinnenbundes durchgeführt werden.

Die Interviews orientierten sich an einem dreigeteilten Leitfaden: Zunächst wurden Fragen zur persönlichen Entwicklung (Kindheit, Jugend, Schule und Berufswahl) gestellt, wobei es auch um die Erfassung von in dieser Zeit vermittelten Werten und Grundhaltungen ging. Der Schwerpunkt des Leitfadens lag jedoch auf der Erfassung der persönlich erlebten Verbandsgeschichte und der eigenen Tätigkeiten und Erfahrungen der Zeitzeugin im djb, wobei zeitlich der Rahmen etwa auf die Jahre zwischen 1950 bis 1990 begrenzt wurde. Abschließend ging es um die Erfassung der Beziehungen und Einflüsse zwischen der beruflichen Arbeit und der Verbandsarbeit.

Der Leitfaden diente bei den Interviews als Orientierung, es blieb aber der Zeitzeugin überlassen, eigene Aspekte und Themen einzubringen, eigene Schwerpunkte zu setzen, die eventuell sogar über die Stichworte des Leitfadens hinausgingen. Diese Vorgehensweise bot Raum für sehr individuelle, durch die jeweilige Persönlichkeit der Befragten geprägte Aussagen. Durch die Erfassung der persönlichen Entwicklung und damit auch die Darstellung von Erfahrungen und Werten und deren Einfluss auf die beruflichen wie verbandlichen Tätigkeiten können Entwicklungslinien in den Blick genommen werden, die in dieser Form durch andere Quellen nicht möglich sind. Der Wert der Interviews für die historische Forschung liegt, wie schon eingangs erwähnt, sicher nicht in der Erfassung von historisch exaktem Faktenmaterial, denn dazu sind Erinnerungen in der Regel zu ungenau, sondern in der Erfassung von persönlichen Werten, Grundhaltungen, Erfahrungswissen und in der Schilderung von persönlichen Beziehungen und Erlebnissen, die in Protokollen und offiziellen Mitteilungen nicht wiedergegeben werden. Damit bietet das Interview als historische Quelle erhellendes Hintergrundwissen zum Beispiel zur Erklärung und Deutung von Entscheidungsprozessen innerhalb des Juristinnenbundes.

\section{Die Interviewpartnerinnen}

Bei der Gruppe der acht ausgewählten Zeitzeuginnen fällt die räumliche Verteilung auf: Alle arbeiteten eher im nordwestdeutschen Raum und in Berlin. Die Altersstruktur der Befragten ist relativ einheitlich: Sechs Frauen sind zwischen 1932 und 1936 geboren, etwas heraus fallen nur die älteste Befragte, Jahrgang 1922, und die jüngste, Jahrgang 1949.

Von den Zeitzeuginnen waren vier als Rechtsanwältinnen tätig, vier als Richterinnen. Darüber hinaus arbeiteten bzw. arbeiten einige zeitweilig im diplomatischen Dienst, als Justizministerin, im Bundesjustizministerium oder haben die wissenschaftliche Laufbahn eingeschlagen. ${ }^{1}$

\footnotetext{
1 Die Aufzählung von zwölf Berufsfeldern bei acht Interviewten ist dadurch zu erklären, dass drei Zeitzeuginnen in verschiedenen Berufsfeldern tätig waren.
} 
Die Länge der Interviews schwankt enorm, nämlich zwischen rund einer Stunde und sechs Stunden Länge, wobei sechs der Interviews zwischen 70 und 115 Minuten Länge liegen. Eine Ausnahme bilden zwei umfassende Interviews (zusammen über neun Stunden) mit einer der Zeitzeuginnen. Insgesamt wurden rund 1173 Min. (d.h. 19 Std. 33 Min.) Interviewzeit aufgenommen und transkribiert, also verschriftlicht. Die Länge der Aufnahmezeit ist, mit Ausnahme des besonders langen Interviews, ähnlich wie in den anderen drei Verbänden.

Innerhalb des Juristinnenbundes waren die Frauen in der Regel Mitglieder in Fachkommissionen (z.B. Rentenrechtskommission, Familienrechtskommission). Vier von ihnen haben als erste Vorsitzende die Arbeit des Juristinnenbundes vorangetrieben. Nur eine der Interviewten ist langjähriges Mitglied, ohne selbst im Verband aktiv geworden zu sein.

Mit Blick auf die thematische Ausrichtung setzten die beteiligten Juristinnen sehr unterschiedliche Schwerpunkte, je nach eigenem Blickwinkel, beruflicher Stellung und dem Engagement im Verband. So gaben einige der Interviewten ihre persönliche Lebensgeschichte eher kurz und an Daten und Fakten orientiert wieder, ohne eine enge Verbindung zum späteren Beruf zu knüpfen, andere banden ihre Entwicklung in zeitgeschichtliche Strömungen und Themen ein und stellten eine enge Verbindung zwischen ihrer persönlichen, der beruflichen und der Verbandsentwicklung her.

Insgesamt zeigte sich, dass diese exemplarischen Interviews einen guten Einblick in die Spannbreite der Sichtweisen auf die Verbandsarbeit, dortige Arbeitsformen und Herangehensweisen und die Bedeutung des Verbandes für einzelne Juristinnen bieten.

Die Bereitschaft, über die persönliche Entwicklung und die eigene Sozialisation zu erzählen, war sehr unterschiedlich ausgeprägt und reichte von einer dichten Verflechtung dieses Hintergrunds mit der späteren beruflichen Orientierung und dem sozialem Engagement bis hin zur sachlich-knappen, nur an Daten orientierten Auflistung, bei der eine Verbindung mit der späteren beruflichen und weltanschaulichen Orientierung nicht aufgezeigt wurde. Insgesamt ist ein sehr individueller Umgang mit der Bedeutung der eigenen Biografie ersichtlich.

Eine Besonderheit im Umgang mit den Interviews - im Vergleich zu den Interviewten der anderen Verbände - ist, dass fast alle Zeitzeuginnen das transkribierte Interview gegenlesen wollten und in der Regel mit vielen sprachlichen und zum Teil auch inhaltlichen Änderungen versehen haben. Nur in einem Fall entschied sich die Juristin von vornherein gegen das Korrekturlesen, da sie selbst an einem Forschungsprojekt mit Interviews gearbeitet hatte und um die Problematik der sprachlichen Besonderheiten von Interviewtranskriptionen wusste.

Diese nun vorgenommenen Veränderungen der ursprünglichen Interviews führen zu einer besonderen Problematik: Ursprünglich war geplant, allen Verbandsarchiven die digitale Hörversion und ergänzend eine Transkription der Interviews ihrer Mitglieder zur Verfügung zu stellen, um den damit arbeitenden WissenschaftlerInnen die Auswertung zu erleichtern und z.B. die Schreibweise von Eigennamen etc. zu bieten.
Durch die starken Veränderungen an den Texten weichen nun die Hörversionen von den überarbeiteten Transkriptionen stark $a b$ und sind nicht mehr zusammen verwendbar. Abschließend wird mit den Betreffenden zu klären sein, wie auf lange Sicht mit den Hörversionen zu verfahren ist, also ob auch sie für die Nutzung (nach einer längeren Sperrfrist) freigegeben werden dürfen oder nur die überarbeiteten Texte.

Damit zusammenhängend sind die mit den Zeitzeuginnen abgeschlossenen Nutzungsverträge, die für eine Verwendung der Interviews in den Archiven notwendig sind, zumeist durch Zusätze so stark eingeschränkt worden, dass für die Veröffentlichung einer Auswertung immer der Kontakt mit der jeweiligen Juristin aufgenommen werden muss. Diese Zurückhaltung bei der Freigabe ihrer Interviews hat mit den beruflichen und verbandlichen Tätigkeiten und Erfahrungen der Zeitzeuginnen zu tun: In der Regel im Schlaglicht der Medien stehend, wissen sie um die Gefahr der unkontrollierten Verwendung von Interviews und sichern sich durch diese Regelung ab. Die Interpretation von sprachlichen Ungenauigkeiten könnte zu Missverständnissen und Unannehmlichkeiten führen, die sie somit verhindern können. So waren alle Juristinnen bei der Nennung von problematischen Situationen und Konflikten innerhalb des Verbandes sehr zurückhaltend. Für die Erforschung von internen Entwicklungssträngen sind Themen wie zum Beispiel Diskussionen über Führungsstile, Bevorzugung und Ablehnung von Arbeitsschwerpunkten des Verbandes sehr interessant. Das Sprechen über Konflikte und Auseinandersetzungen birgt allerdings die Gefahr, dass neue Konflikte durch die Nennung von Namen hervorgerufen werden oder insgesamt Bedenken bestehen, dem Ansehen des Verbandes zu schaden. Daher ist die Schilderung von schwierigen Situationen in Verbänden immer eine Gratwanderung. Für die Forschung dagegen sind dies oftmals wertvolle Schilderungen, weil sie Erklärungen liefern können für bestimmte Entwicklungen.

Für die Erforschung der Geschichte des djb bieten die Interviews dennoch vielfältige Aspekte, zum Beispiel werden in ihnen die Erfahrungen und Grundhaltungen aufgezeigt, aus denen einzelne Frauen handeln. Sie zeigen auch, welche Auswirkung die Arbeit im Verband auf die persönliche und berufliche Entwicklung der Frauen hatte, beispielsweise die enorme Motivation, die durch erfolgreiche Aktionen entstand, aber auch die Unterstützung, die sie erfuhren durch den gegenseitig gebotenen Rückhalt und das „Netzwerken“ hinter den Kulissen.

An dieser Stelle soll Frau Gimbal und Frau Bosch ganz herzlich für die freundliche Vermittlung von Zeitzeuginnen gedankt werden. Ein großes Dankeschön geht aber vor allem an die Zeitzeuginnen selbst, die sich bereit erklärt haben, sich den umfassenden Fragen und Themen zu stellen.

\section{Wie weiter?}

Wie anfangs erwähnt hat das Projekt das Ziel, zur Bewahrung von erzählter Geschichte anzuregen, das heißt Frauenverbänden aus den jetzt gemachten Erfahrungen heraus Informationen über Möglichkeiten und Wege zu vermitteln, wie sie dies 
selbst organisieren können. In der letzten Projektphase bis Februar 2009 wird deshalb eine Internetpräsentation erstellt, die Hinweise und Tipps zur Durchführung von Interviews geben soll. Dort werden Themen wie Interviewtechniken, Transkriptionsregeln, die erforderliche technische Ausrüstung, rechtliche Aspekte, Sicherung und Auswertung und vieles mehr erläutert. Dabei wird - ähnlich wie bei den eingangs erwähnten Seiten zu Archivfragen (www.FrauVerA.de) - mit Beispielen und
Links zu weiterführenden Informationen gearbeitet werden. Wir hoffen, damit Frauenvereinen und -verbänden ein Hilfsmittel an die Hand zu geben, das ihnen ermöglicht, selbst in dieser Form die Geschichte ihres Verbandes in „Erzählungen“ zu dokumentieren.

Selbstverständlich stehen wir auch gerne allen Interessierten für Auskünfte zur Verfügung (loeneke@addf-kassel.de oderwenzel@addf-kassel.de).

\section{Deutsche Juristinnen von 1948 bis 1989}

Nachdem Juristinnen während des Regimes des Nationalsozialismus weitgehend aus dem Berufsleben ausgeschlossen gewesen waren, kam ihnen nach 1945 der große Bedarf sowohl in der Anwaltschaft als auch in der Rechtsprechung zu Gute. War ihnen zuvor die Berufsausübung verwehrt worden, konnten Rechtsanwältinnen und Richterinnen nun davon profitieren, als nicht belastet zu gelten. Deutsche Juristinnen wurden mit Verteidigungen vor den Militärgerichten betraut, noch bevor die deutsche Justiz ihre Tätigkeit wieder aufgenommen hatte. Sie wirkten beim Aufbau der kommunalen Verwaltungen mit und wurden Richterinnen an Amts-, Land- und Oberlandesgerichten. Juristinnen fanden sich auch in den politischen Parteien. Viele teilten das Schicksal, ihren Ehepartner im Krieg verloren zu haben. Sie mussten nicht nur ihre Kinder allein erziehen, sondern auch für den Lebensunterhalt einer ganzen Familie sorgen.

Dr. Erna Scheffler, während des NS-Regimes vom Beruf ausgeschlossen, wurde im September 1951 als einzige Frau an das neu gegründete Bundesverfassungsgericht in Karlsruhe berufen. Dr. Gerda Krüger-Nieland und Elisabeth Krumme wurden Richterinnen der ersten Stunde am Bundesgerichtshof, Dr. Anne-Gudrun Meier-Scherling folgte einem Ruf an das Bundesarbeitsgericht, Dr. Maria Schwarz ging an das Bundessozialgericht und Charlotte Schmidt wurde Richterin am Bundesverwaltungsgericht.

Im August 1948 gründeten sieben Juristinnen ${ }^{1}$ in Dortmund wieder einen Verein für Juristinnen, nunmehr auch für Volkswirtinnen. Die „Vereinigung weiblicher Juristen und Volkswirte e.V.“ verstand sich als Nachfolgeorganisation des 1914 gegründeten „Deutschen Juristinnen-Vereins, e.V.“. Die 1. Vorsitzende Hildegard Gethmann aus Dortmund wollte weibliche Kräfte zur Wahrnehmung der Interessen von Frauen im öffentlichen Leben bündeln und verlangte dafür von jeder Frau, die dem Verein beitrat, die hohe Einsatzbereitschaft, die ihr selbst zu eigen war.

\section{Das Ringen um Artikel 3 Abs. 2 und 3 GG}

Die Verankerung der Gleichberechtigung von Frau und Mann in Artikel 3 des Grundgesetzes verlieh dem Anliegen der Frauen erstmals eine rechtliche Grundlage zur Durchsetzung der Gleichberechtigung in allen Rechtsgebieten. Bis es zur entsprechenden Fassung des Artikel 3 GG kam, hatte es im Parlamentarischen Rat einen zähen Machtkampf gegeben, dessen Ausgang im Wesentlichen dem Verhandlungsgeschick der Rechtsanwältin Dr. Elisabeth Selbert zu verdanken ist. Fortschrittlich hinsichtlich einer paritätischen Behandlung von Frau und Mann im Recht schon in ihrer Dissertation 1930, hielt sie für selbstverständlich, dass die Gleichberechtigung im Grundgesetz festgehalten wurde. Als eine von vier Frauen sah sie sich im Parlamentarischen Rat jedoch 61 männlichen Abgeordneten gegenüber, die es indiskutabel fanden, Frauen durch die Verfassung gleiche Rechte im Hinblick auf alle Rechtsgebiete zu gewähren. Dem stetigen Drängen Elisabeth Selberts wurde erst nachgegeben, als Frauen aus der gesamten Republik mit Protestschreiben von den Abgeordneten die volle Gleichberechtigung forderten. Infolgedessen legten Artikel 3 Absatz 2 und Absatz 3 des Grundgesetzes das Recht der Frau auf Gleichberechtigung und ein Verbot der Ungleichbehandlung von Frau und Mann fest. Alle hiermit unvereinbaren Gesetze, so das Grundgesetz weiter, sollten mit Ablauf des 31. Dezember 1953 keine Gültigkeit mehr haben. Das Erarbeiten von Vorschlägen für neue, mit der Verfassung vereinbare Gesetze war

\footnotetext{
1 Rechtsanwältin Hildegard Gethmann, Assessorin Luise Purps, Rechtsanwältin Dr. Ruth Rogalski-Rohwedder, Regierungsrätin Dr. Anna Schlieper, Anwaltsassessorin Alma Schmidt-Perchner, Anwaltsassessorin Annette Schücking und Rechtsanwältin Elisabeth Späth-Uden.
}

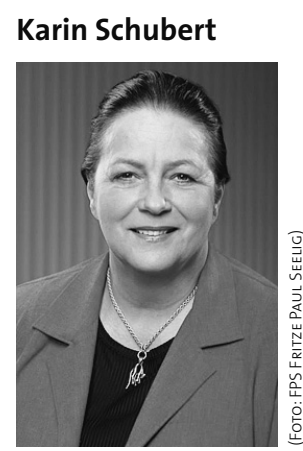

Senatorin a.D., Justizministerin a.D., Rechtsanwältin, Berlin 